\title{
Search for variable stars in the open cluster NGC 2539
}

\author{
K. J. Choo ${ }^{1,2}$, S.-L. Kim ${ }^{2}$, T. S. Yoon ${ }^{1}$, M.-Y. Chun ${ }^{2}$, H. Sung ${ }^{3}$, \\ B.-G. Park ${ }^{2}$, H. B. Ann ${ }^{4}$, M. G. Lee ${ }^{5}$, Y.-B. Jeon ${ }^{2}$, and I.-S. Yuk ${ }^{2}$ \\ 1 Department of Astronomy and Atmospheric Sciences, Kyungpook National University, Daegu 702-701, Korea \\ 2 Korea Astronomy Observatory, Daejeon 305-348, Korea \\ ${ }^{3}$ Department of Astronomy and Space Science, Sejong University, Seoul 143-747, Korea \\ ${ }^{4}$ Department of Earth Science, Pusan National University, Busan 609-735, Korea \\ 5 Astronomy Program, SEES, Seoul National University, Seoul 151-742, Korea
}

Received 17 July 2002 / Accepted 7 November 2002

\begin{abstract}
We present $U B V I$ absolute and $V$-band time-series CCD photometric results for the intermediate-age open cluster NGC 2539. From the empirical zero-age main sequence and theoretical isochrone fitting in color-color and color-magnitude diagrams, we estimated the physical parameters of this cluster as follows: the color excess of $E(B-V)=0.06 \pm 0.03$, the distance modulus of $\left(V-M_{V}\right)_{0}=10.2 \pm 0.1$ and the age of $\log t=8.8(\sim 630 \mathrm{Myr})$. By carefully examining the time-series images, we discovered seven new variable stars in the observed cluster field. Considering the light curves, periods and positions on the color-magnitude diagram, we classified them as five eclipsing binary stars, one $\delta$ Scuti star and one $\gamma$ Doradus candidate. The $\gamma$ Doradus candidate might not be a cluster member because it is located redder by about $\Delta(B-V)=0.2$ than the cool edge of $\gamma$ Doradus instability strip.
\end{abstract}

Key words. open clusters and association: individual: NGC 2539 - stars: Hertzsprung-Russell (HR) and C-M diagrams stars: binaries: eclipsing

\section{Introduction}

$\delta$ Scuti and $\gamma$ Doradus type pulsating stars are very important objects for the investigation of the internal structure and evolution theory of intermediate-mass stars from the asteroseismological approach (see Breger 2000 and Zerbi 2000 for recent reviews). $\delta$ Scuti stars are main sequence stars of spectral types from A3 to F0, having short pulsational periods of 0.02 to 0.3 day and solar metal abundance. They lie on or above the main sequence facing on an extension of Cepheid instability strip in the Hertzsprung-Russell diagram. Their pulsational characteristics show slight differences according to their evolution stages (Breger 1991). Stars in the hydrogen shell burning stage have generally one or two periods with pure radial modes and high amplitudes of more than 0 . 1 . In comparison with these high amplitude $\delta$ Scuti stars (so called, HADS), stars in the hydrogen core burning stage near the zero-age main sequence have multi-frequencies with radial modes as well as non-radial ones and small amplitudes of several millimagnitude.

$\gamma$ Doradus stars are early F-type main sequence or subgiant stars which have longer periods of $0.4 \sim 3.0$ day than that

Send offprint requests to: K. J. Choo, e-mail: kjchoo@knu.ac.kr of $\delta$ Scuti stars and amplitudes of less than 0 . 1 in $V$-band. Although the variability of $\gamma$ Doradus itself was discovered about 40 years ago by Cousins \& Warren (1963), $\gamma$ Doradus type stars have been only recently classified as a new type of pulsating variable. The reason is that only a few stars with similar characteristics have been known and their variability was not understood theoretically until a few years ago. They are located around the cool edge of $\delta$ Scuti instability strip (Kaye et al. 1999). Based on the Hipparcos photometry data, Handler (1999) defined the $\gamma$ Doradus instability strip as ranges of $7200 \sim 7700 \mathrm{~K}$ on the zero-age main sequence and of $6900 \sim 7500 \mathrm{~K}$ with one magnitude brighter than it. They are thought to be excited in high-order $(n)$ low-degree $(l)$ non-radial gravity-modes.

A number of pulsating stars such as $\delta$ Scuti, $\gamma$ Doradus, $\beta$ Cephei and slowly pulsating B-type stars were found in young- or intermediate-age open clusters. Frandsen \& Arentoft (1998) discovered seven $\delta$ Scuti stars in NGC 1817 and Freyhammer et al. (2001) found $13 \delta$ Scuti stars in the central field of NGC 7062. Especially, Arentoft et al. (2001) detected 17 new variable stars in NGC 6231, including three $\delta$ Scuti stars, three $\gamma$ Doradus star candidates, three slowly pulsating B-type star candidates and two $\beta$ Cephei stars. One very good recent review article for this topic is the paper by 
Rodríguez \& Breger (2001). Using $\delta$ Scuti stars in open clusters, Suarez et al. (2002) investigated correlation between the oscillation amplitudes and stellar parameters of $\delta$ Scuti stars.

This study is part of the photometric survey of variable stars in open clusters, which has been carried out at the Bohyunsan Optical Astronomy Observatory (BOAO) in Korea. General descriptions and the most recent results for the survey are given in Kim et al. (2001a, b). We selected an intermediate-age open cluster NGC $2539\left(\mathrm{RA}_{2000}=8^{\mathrm{h}} 10^{\mathrm{m}} 42^{\mathrm{s}}, \operatorname{Dec}_{2000}=-12^{\circ} 50^{\prime}\right)$ at the constellation Puppis in the southern sky. This cluster is classified as II $1 \mathrm{~m}$ type (Ruprecht 1966), in which stars are moderately concentrated. The cluster is appropriate for our less-wide CCD camera. It has a lot of A $\sim \mathrm{F}$ type main sequence stars which are thought to be $\delta$ Scuti and $\gamma$ Doradus candidates. The observations and data reduction are described in Sect. 2. We present physical parameters of this cluster and properties of newly detected variable stars in Sect. 3.

\section{Observations and data reduction}

The observations were performed for ten nights between February and March, 2000 at the BOAO. We used $1.8 \mathrm{~m}$ telescope and a SITe $2048 \times 2048$ CCD camera. The gain and readout noise of the CCD camera are $1.8 \mathrm{e}^{-} / \mathrm{ADU}$ and $7.0 \mathrm{e}^{-}$, respectively. The field of view is about $11.6 \times 111^{\prime} 6$ with the scale of 0 ! $34 /$ pixel. In order to estimate physical parameters of NGC 2539, we carried out $U B V I$ CCD photometry in a clear night on February 10, 2000. Johnson $U B V$ and Cousins $I$ filters were used. For the purpose of obtaining standard magnitudes and colors of stars in the cluster region, $U B V R I$ standard star fields of SA $98\left(\mathrm{RA}_{2000}=6^{\mathrm{h}} 52^{\mathrm{m}} 22^{\mathrm{s}} \cdot 5, \mathrm{Dec}_{2000}=-0^{\circ} 19^{\prime} 6^{\prime \prime}\right)$ and SA $99\left(\mathrm{RA}_{2000}=7^{\mathrm{h}} 56^{\mathrm{m}} 0.5, \operatorname{Dec}_{2000}=-0^{\circ} 18^{\prime} 48^{\prime \prime}\right)$ by Landolt (1973) were also observed.

We performed time-series photometry to search for variable stars in this cluster. A total of 581 time-series CCD frames were obtained with the $V$ filter. The typical photometric seeing $(F W H M)$ was about 2'. 0 . Exposure times were adjusted in a range from 18 to $30 \mathrm{~s}$, depending on seeing and transparency. In order to minimize position-dependent external errors (Frandsen et al. 1989), we carefully controlled the telescope by locating a star at the same position in the CCD frames during our observing run. Figure 1 represents the observed CCD field of NGC 2539 and the detailed observation log is listed in Table 1.

All CCD images were preprocessed to correct overscan, trim unreliable and useless regions, subtract bias frames, correct flat fielding and reject cosmic rays using the IRAF/CCDRED package. Then, we obtained instrumental magnitudes of stars from the empirical point spread function fitting method of the IRAF/DAOPHOT package (Massey \& Davis 1992). After taking into account the differences of aperture radii between standard stars $\left(\sim 7^{\prime \prime}\right)$ and stars in the dense cluster area (1 FWHM), we derived the following standard transformation equations:

$$
\begin{aligned}
& U=u+19.616+0.359(B-V)-0.488 X, \sigma_{U}=0.074 \\
& B=b+22.597+0.173(B-V)-0.226 X, \sigma_{B}=0.045 \\
& V=v+23.160+0.020(B-V)-0.097 X, \sigma_{V}=0.043 \\
& I=i+23.335+0.034(V-I)-0.057 X, \sigma_{I}=0.025
\end{aligned}
$$

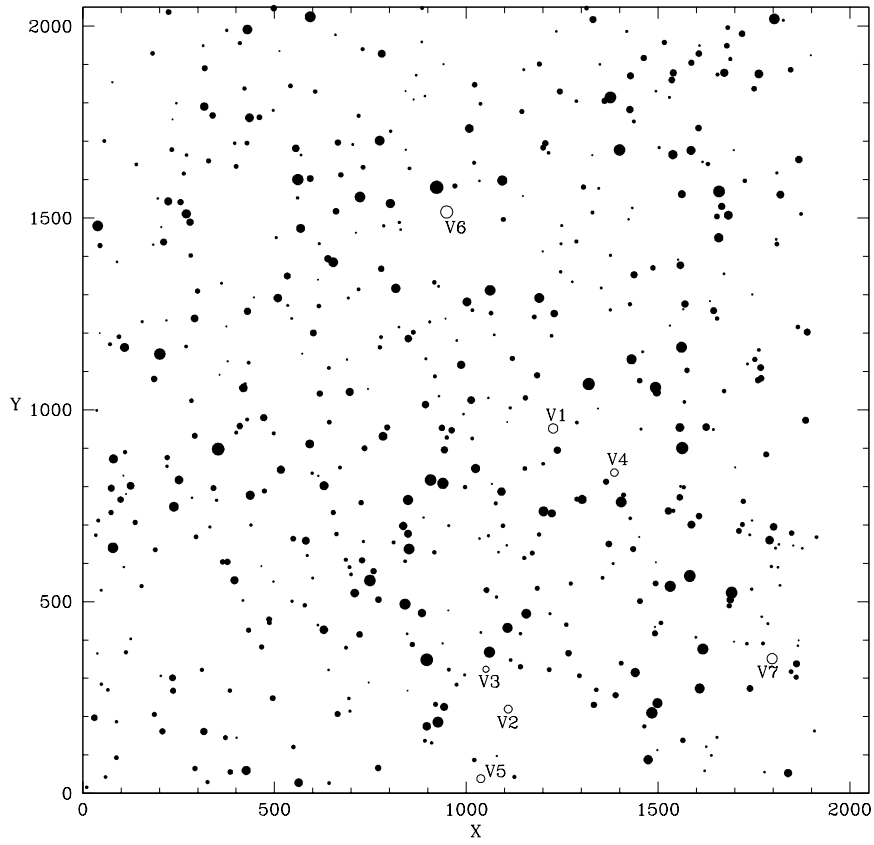

Fig. 1. Observed CCD field $(11: 6 \times 11: 6)$ of the open cluster

\begin{tabular}{|c|c|c|c|c|}
\hline $\begin{array}{l}\text { Date } \\
(2000)\end{array}$ & $\begin{array}{l}\text { Start } \\
\text { HJD }\end{array}$ & $\begin{array}{l}\text { Run } \\
\text { time }\end{array}$ & $\begin{array}{l}\text { No. of } \\
\text { images }\end{array}$ & $\begin{array}{c}\text { Observation } \\
\text { mode }^{\dagger}\end{array}$ \\
\hline Feb. 9 & $2451584^{\mathrm{d}} .02$ & $2 \cdot 0$ & 55 & $\mathrm{TS}(V)$ \\
\hline Feb. 10 & $2451584^{d} .99$ & $2 \mathrm{~h} .2$ & 42 & $\begin{array}{l}\text { TS }(V) \text {, Short-Long } \\
\text { STD }(U B V I)\end{array}$ \\
\hline Feb. 16 & $2451590 \cdot 99$ & $2 \mathrm{~h} .0$ & 44 & $\operatorname{TS}(V)$ \\
\hline Feb. 20 & $2451594^{\mathrm{d}} \cdot 98$ & $2 \cdot 0$ & 33 & $\mathrm{TS}(V)$ \\
\hline Feb. 21 & 2451596.02 & 3.0 & 47 & $\mathrm{TS}(V)$ \\
\hline Feb. 22 & 2451596.95 & 5.0 & 124 & $\mathrm{TS}(V)$ \\
\hline Mar. 14 & 2451617.94 & $4^{\mathrm{h}} .5$ & 116 & $\mathrm{TS}(V)$ \\
\hline Mar. 24 & 2451628.02 & 0.5 & 17 & $\mathrm{TS}(V)$ \\
\hline Mar. 25 & 2451629.00 & 2.0 & 41 & $\mathrm{TS}(V)$ \\
\hline \multirow[t]{2}{*}{ Mar. 26} & $2451630^{\mathrm{d}} .00$ & $2^{\mathrm{h}} \cdot 0$ & 62 & $\mathrm{TS}(V)$ \\
\hline & 10 nights & $25^{\mathrm{h}} .2$ & 581 & \\
\hline
\end{tabular}
NGC 2539. Seven new variable stars are represented by open circles with their IDs. The size of the symbol is proportional to the brightness.

Table 1. Observation log.

$\mathrm{TS}(V): V$ filter time-series.

Short: short-exposed images of NGC 2539 $(U: 120 \mathrm{~s}, B: 30 \mathrm{~s}, V: 25 \mathrm{~s}, I: 5 \mathrm{~s})$.

Long: long-exposed images of NGC 2539 $(U: 480 \mathrm{~s}, B: 360 \mathrm{~s}, V: 180 \mathrm{~s}, I: 30 \mathrm{~s})$.

STD: standard stars in the SA 98 and SA 99 regions.

where $U, B, V$ and $I$ are standard magnitudes, and $u, b, v$ and $i$ are instrumental magnitudes. The airmass is denoted as $X$. Quantum efficiency of the BOAO CCD camera is sharply declined in $U$ filter, resulting in large photometric errors and large dispersions near $(U-B)=0.0$. Therefore, we used the color term $(B-V)$ instead of $(U-B)$ in the transformation equation for $U$ filter (Sung et al. 2001).

We obtained standard magnitudes and colors of stars in the observed field of NGC 2539 from the above equations, 

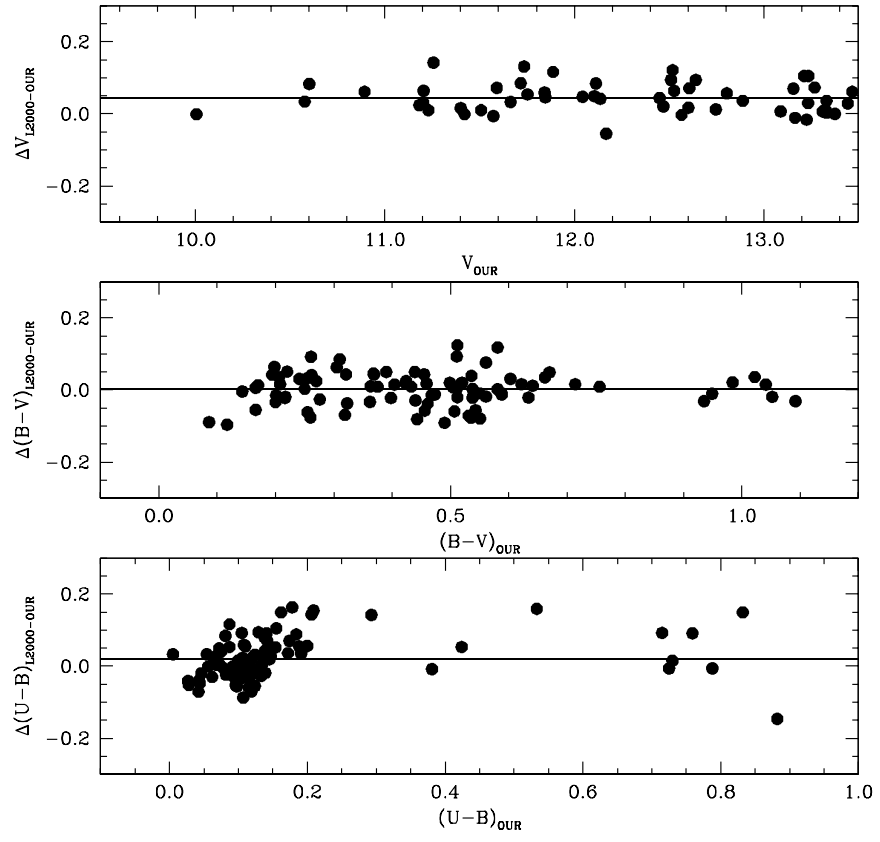

Fig. 2. Magnitude and color differences between our photometric results and the previous data by Lapasset et al. (2000).

and compared our $U B V I \mathrm{CCD}$ photometric results with the previous photoelectric data; by Pesch (1961), $\Delta V_{\mathrm{P} 1961-\text { OURS }}=$ $+0.028 \pm 0.050, \Delta(B-V)_{\mathrm{P} 1961-\mathrm{OURS}}=+0.019 \pm 0.037$ and $\Delta(U-B)_{\mathrm{P} 1961-\mathrm{OURS}}=+0.004 \pm 0.033$, by Joshi \& Sagar (1986), $\Delta V_{\text {JS1986-OURS }}=+0.045 \pm 0.065, \Delta(B-V)_{\text {JS1986-OURS }}=$ $+0.000 \pm 0.055$ and $\Delta(U-B)_{\mathrm{JS} 1986-\mathrm{OURS}}=-0.034 \pm 0.021$, by Lapasset et al. (2000), $\Delta V_{\text {L2000-OURS }}=+0.044 \pm 0.043$, $\Delta(B-V)_{\mathrm{L} 2000-\mathrm{OURS}}=+0.003 \pm 0.047$ and $\Delta(U-B)_{\mathrm{L} 2000-\mathrm{OURS}}=$ $+0.020 \pm 0.062$ (Fig. 2). Our $V$ magnitudes are a little brighter by about -0.035 than the previous results, but our color indices are in good agreement with them.

For standardization of time-series images, we adopted an ensemble normalization technique (Gilliland \& Brown 1988). To begin with, we chose tens of normalization stars which are bright and unsaturated stars from 11 mag to $13 \mathrm{mag}$, except for peculiar stars such as variables or stars near the CCD edge. The coefficients in the following equation were derived for each time-series frame:

$V=v+a_{1}+a_{2}(B-V)+a_{3} X+a_{4} Y$

where $V$ and $v$ are the standard and the instrumental magnitudes of normalization stars, respectively. $X$ and $Y$ denote the position of a star in the CCD frame. $a_{1}$ and $a_{2}$ are the zero point and the color coefficients, $a_{3}$ and $a_{4}$ the position coefficients of $X$ - and $Y$-axis. The coefficients depend on the atmospheric extinction and the instrumental condition of each night. Then, we obtained standard magnitudes of all stars in each time-series frame using the above equation.

\section{Results}

\subsection{Physical parameters of NGC 2539}

We present the color-color diagram (Fig. 3) and the colormagnitude diagrams (Fig. 4) from the $U B V I$ photometric data.

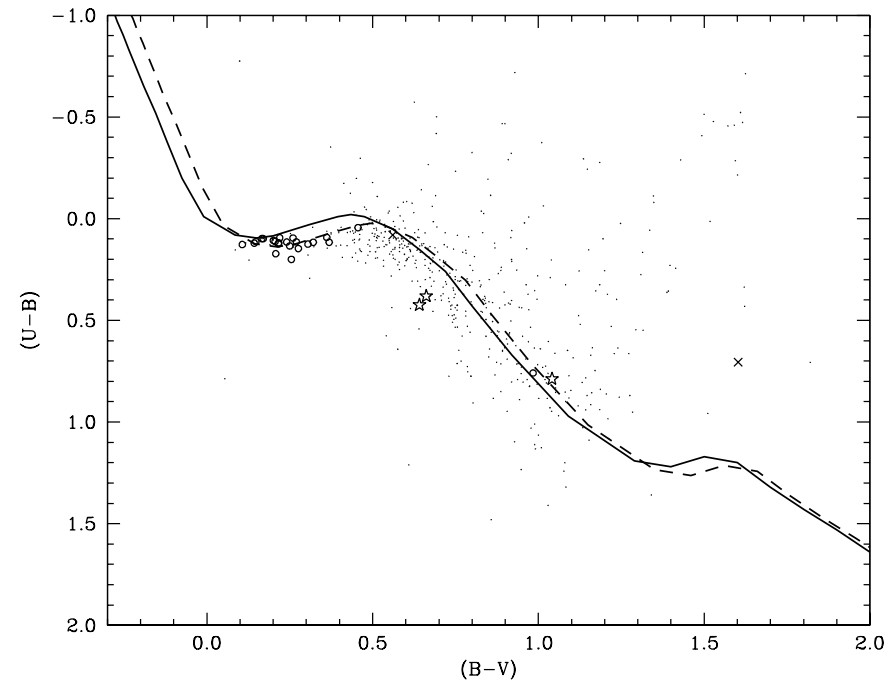

Fig. 3. Color-color diagram of the open cluster NGC 2539. Solid and dashed lines denote the unreddened and reddened empirical ZAMS by Sung \& Bessell (1999), respectively. Open circles: cluster members, star symbols: spectroscopic binaries and crosses: non-members, classified by Joshi \& Sagar (1986), Clariá \& Lapasset (1986) and Mermilliod \& Mayor (1989). Dots are stars with unknown membership.

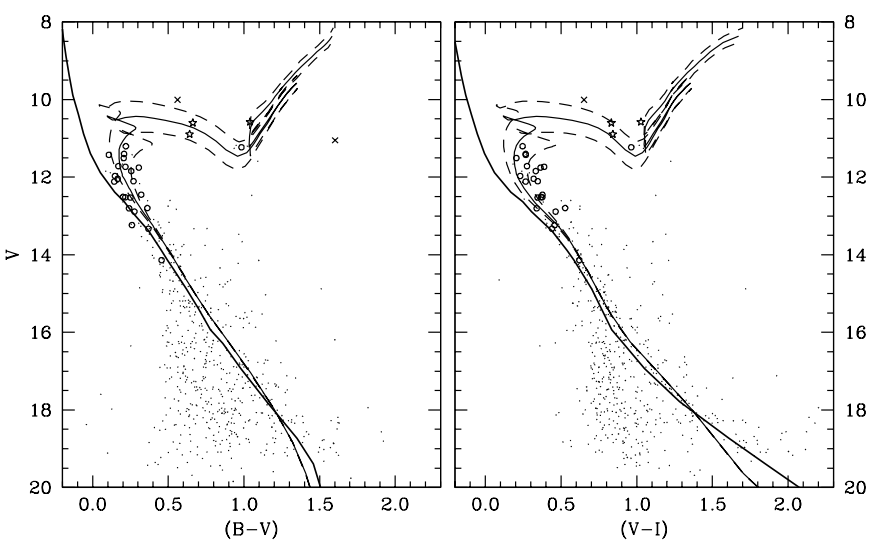

Fig. 4. Color-magnitude diagrams of the open cluster NGC 2539. Thick lines denote the empirical ZAMS by Sung \& Bessell (1999). Thin and dashed lines represent the theoretical isochrones for $Z=0.019$ by Girardi et al. (2000). From upper to the lower, the isochrones represent $\log t(\mathrm{yr})=8.7,8.8$ and 8.9 , respectively. Symbols are the same as in Fig. 3.

In order to determine more accurate physical parameters of this cluster, we used cluster members with high priorities. Joshi \& Sagar (1986) identified cluster members based on their positions in the color-color and color-magnitude diagrams and their spatial distributions in the sky. Clariá \& Lapasset (1986) judged memberships of a few red giants by examining interstellar extinctions and luminosity classes, and Mermilliod \& Mayor (1989) by measuring radial velocities. We estimated the interstellar reddening value $E(B-V)=0.06 \pm 0.03$ from the empirical zero-age main sequence fitting in the color-color diagram, with the assumption of $E(U-B) / E(B-V)=0.72$. Another reddening value $E(V-I)=0.08 \pm 0.03$ was calculated by the adoption of $E(V-I) / E(B-V)=1.25$ (Dean et al. 1978). 

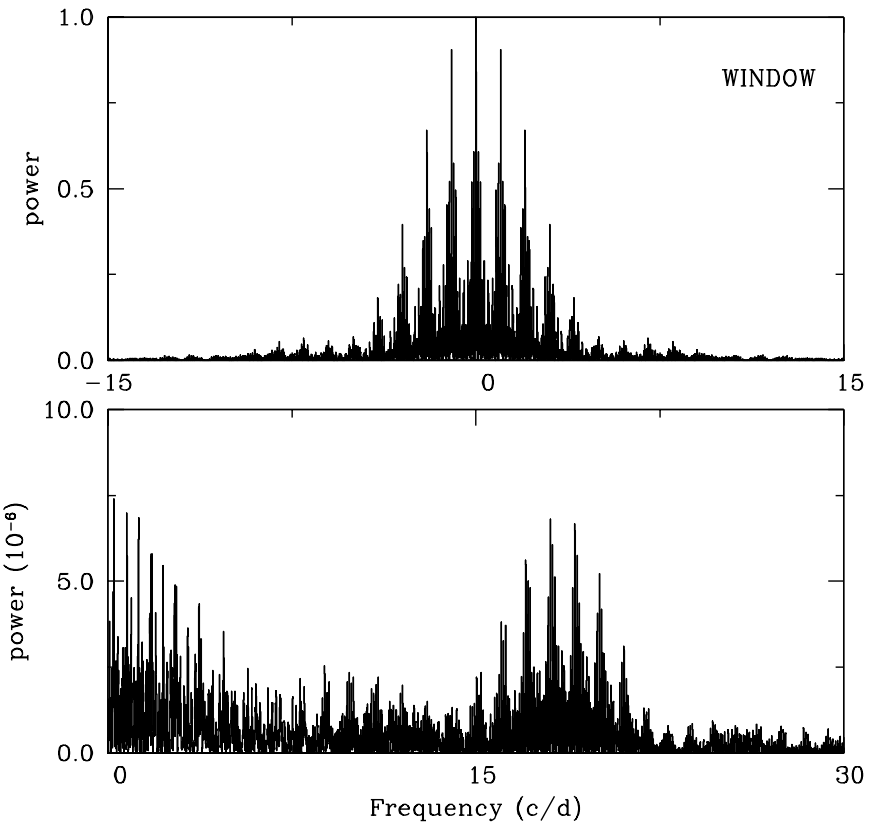

Fig. 5. Power spectra of $V 1$. The upper panel shows spectral window. The dominant pulsating frequency of $V 1$ is 18.032 cycles/day.

Solid and dashed lines in Fig. 3 denote the unreddened and reddened zero-age main sequences by Sung \& Bessell (1999) for solar metal abundance.

We determined the distance modulus of $\left(V-M_{V}\right)_{0}=$ $10.2 \pm 0.1$ and the cluster age of $\log t(\mathrm{yr})=8.8$ from the zero-age main sequence and theoretical isochrone fitting in two color-magnitude diagrams of $(B-V)$ vs. $V$ and $(V-I)$ vs. $V$ as shown in Fig. 4. We assumed the total extinction value of $A_{V}=3.1 \times E(B-V)$ and made use of the theoretical isochrones with $Z=0.019$ calculated by Girardi et al. (2000).

In Table 2, we compare our results with the previous ones. Our values are generally similar to other ones; especially they are in good agreement with the recent values by Lapasset et al. (2000).

\subsection{Variable stars in NGC 2539}

We examined light variations of 583 stars using a total of 581 time-series CCD frames. Seven new variable stars were discovered. Position of variable stars, which are arbitrarily labeled from $V 1$ to $V 7$, are marked as open circles in Fig. 1. Two of them, $V 1$ and $V 2$, were identified as pulsating stars from their light curves. We estimated pulsating periods of $V 1$ using the multiple frequency analysis by Kim \& Lee (1996). Figures 5 and 6 show the power spectra and light curves of $V 1$, respectively. From the bottom panel of Fig. 5, we detected a pulsation frequency of $f_{1}=18.032$ cycles/day ( 0.055 day). Another frequency of $f_{2}=0.240$ cycles/day seems to be caused by daily variations, maybe the flat-field problem of our observation system (Sung et al. 2001); this might be the reason that a little large dispersions are shown in phase diagrams of $V 2 \sim V 7$ (Fig. 7). The period of another pulsating variable $V 2$ was determined to be 0.352 from the phase-match technique. Its light curve is shown in Fig. 7 together with five eclipsing
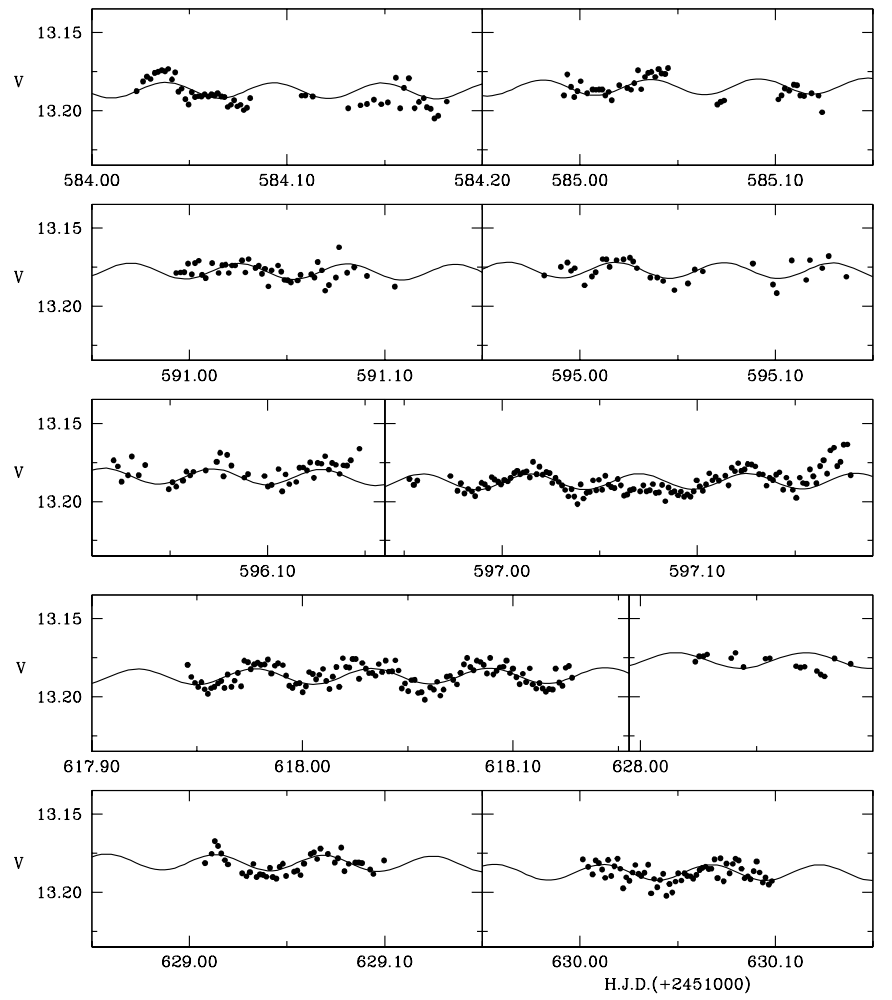

Fig. 6. Light variations of $V 1$. The solid lines represent synthetic curves made by amplitudes and phases of two frequencies, $f_{1}=$ $18.032 \mathrm{cycles} /$ day and $f_{2}=0.240$ cycles/day.

binary stars from $V 3$ to $V 7$. Positions of two pulsating variables in the color-magnitude diagram are marked by star symbols in Fig. 8. Considering the positions, pulsating periods and amplitudes, we classify $V 1$ as a $\delta$ Scuti star and $V 2$ as a $\gamma$ Doradus star. But it should be noted that $V 2$ might be a field star having larger reddening value of $\Delta E(B-V) \geq 0.2$ than the cluster members, because this star is cooler and fainter than the cool edge of $\gamma$ Doradus instability strip (Handler 1999).

The light curves of five eclipsing binary stars $V 3 \sim V 7$ are shown in Fig. 7. These stars are represented by open circles in the color-magnitude diagram of Fig. 8. V5 shows the primary and secondary minima which are clearly discernible in spite of large dispersions of data points in the out-of-eclipse phase. Considering the position of $V 6$ in the color-magnitude diagram, we think that $V 6$ is a field star, as Clariá \& Lapasset (1986) insisted. Basic parameters of seven new variable stars are summarized in Table 3.

Until a few years ago, $\gamma$ Doradus stars were considered to be young objects from the fact that they were discovered in several open clusters with ages less than $250 \mathrm{Myr}$ such as NGC 2516 (age 137 Myr, Zerbi et al. 1998), M 34 (age 250 Myr, Krisciunas \& Patten 1999), M 45 (age 74 Myr, Martín \& Rodríguez 2000) and NGC 2301 (age $\sim 250 \mathrm{Myr}$, Kim et al. 2001b). No $\gamma$ Doradus candidates were detected in the Hyades cluster with age of about $600 \mathrm{Myr}$ (Krisciunas et al. 1995). Our result, no $\gamma$ Doradus stars in NGC 2539 with the cluster age of $630 \mathrm{Myr}$, is consistent with this suggestion by Krisciunas \& Patten (1999). However, recent photometric survey discovered a few $\gamma$ Doradus 
Table 2. Physical parameters of the open cluster NGC 2539.

\begin{tabular}{ccccc}
\hline \hline Reference & $E(B-V)$ & $\left(V-M_{V}\right)_{0}$ & $\operatorname{Age}\left(\times 10^{8} \mathrm{yr}\right)$ & {$[\mathrm{Fe} / \mathrm{H}]$} \\
\hline Pesch (1961) & $0.10 \pm 0.05$ & 10.6 & - & - \\
Clariá \& Lapasset (1986) & $0.08 \pm 0.02$ & $9.8 \pm 0.5$ & $6.4 \pm 0.8$ & $(+0.24 \pm 0.06)_{\mathrm{DDO}}$ \\
& & & & $(+0.2 \pm 0.1)_{\mathrm{CM}}$ \\
& & & & $(-0.2 \pm 0.1)_{\mathrm{MT}_{1}}$ \\
Joshi \& Sagar (1986) & $0.08 \pm 0.02$ & $10.1 \pm 0.3$ & 5.4 & - \\
Lapasset et al. (2000) & 0.06 & 10.42 & 6.3 & - \\
Our study & $0.06 \pm 0.03$ & $10.2 \pm 0.1$ & 6.3 & - \\
\hline
\end{tabular}
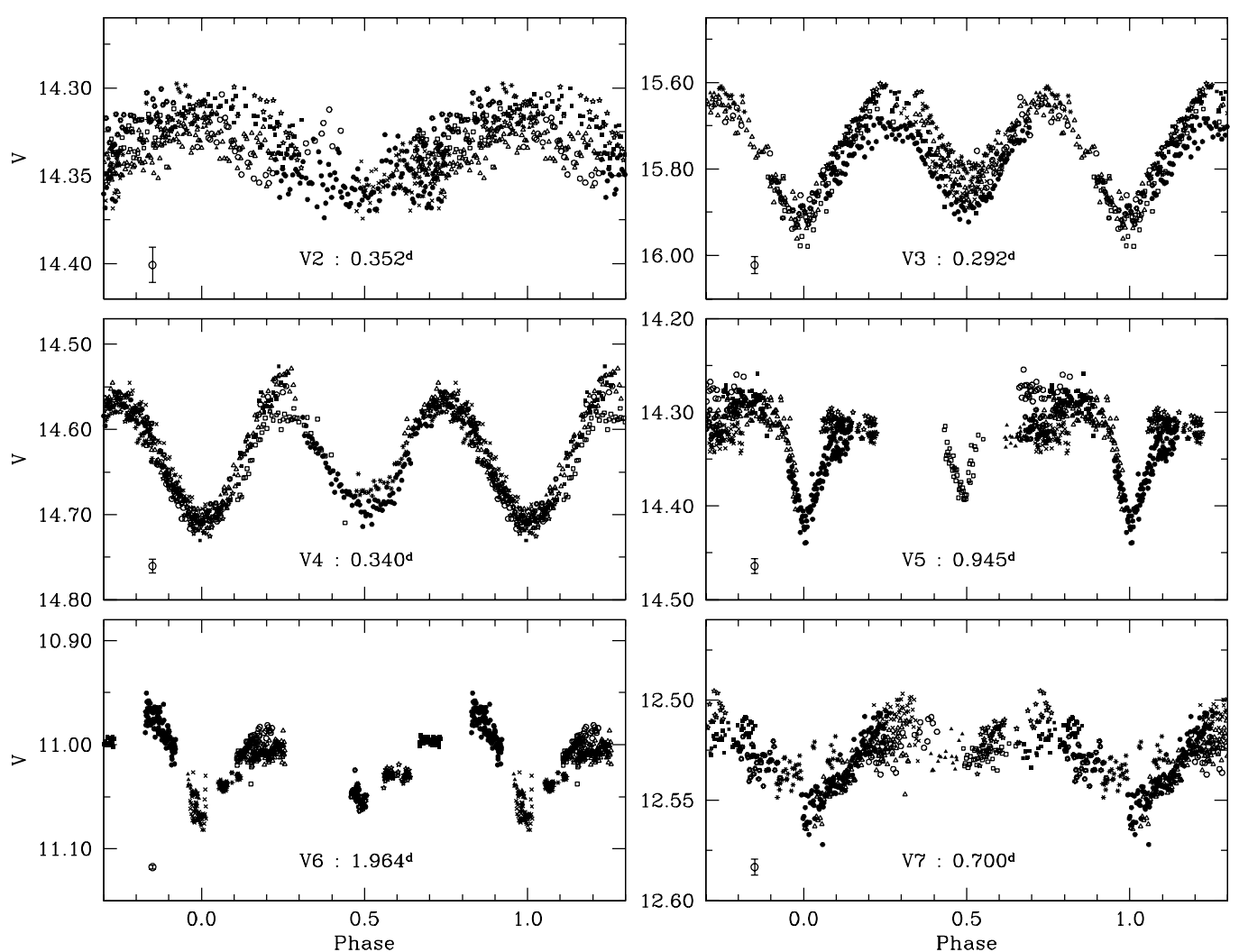

\begin{tabular}{|lllll}
\hline « $09-\mathrm{Feb}-2000$ & $* 10-\mathrm{Feb}-2000$ & व $16-\mathrm{Feb}-2000$ & $020-\mathrm{Feb}-2000$ & - $21-\mathrm{Feb}-2000$ \\
$\Delta 22-\mathrm{Feb}-2000$ & $\bullet 14-\mathrm{Mar}-2000$ & $\Delta 24-\mathrm{Mar}-2000$ & $* 25-\mathrm{Mar}-2000$ & $\times 26-\mathrm{Mar}-2000$
\end{tabular}

Fig. 7. Light curves of $V 2$ to $V 7$. Data points are differently marked for each observing night. Mean error bars are shown at the left bottom in each panel.

Table 3. Basic parameters of seven new variable stars in the open cluster NGC 2539.

\begin{tabular}{|c|c|c|c|c|c|c|c|c|}
\hline ID & $\mathrm{ID}_{\mathrm{BDA}}$ & $\langle V\rangle$ & $B-V$ & Period & $\Delta V_{\max }$ & Epoch & Type & membership \\
\hline$V 1$ & 113 & 13.213 & 0.255 & 0.055 & $\sim 0.02$ & 2451597.1575 & $\delta$ Scuti & - \\
\hline$V 2$ & - & 14.290 & 0.577 & 0.352 & $\sim 0.04$ & 2451585.13 & $\gamma$ Doradus & field (?) \\
\hline$V 3$ & - & $15^{\mathrm{m}} \cdot 652$ & 0.739 & 0.292 & $\sim 0.25$ & 2451591.025 & Eclipsing binary & - \\
\hline$V 4$ & - & 14.584 & 0.749 & 0.340 & $\sim 0.13$ & 2451596.06 & Eclipsing binary & - \\
\hline$V 5$ & 329 & $14^{\mathrm{m}} \cdot 301$ & 0.581 & 0.945 & $\sim 0.13$ & 2451617.99 & Eclipsing binary & - \\
\hline$V 6$ & 133 & $11^{\mathrm{m}} .050$ & 1. 603 & 1.964 & $\sim 0.07$ & 2451630.07 & Eclipsing binary & field \\
\hline$V 7$ & 338 & $12 \mathrm{~m} 527$ & 0.250 & 0.700 & $\sim 0.04$ & 2451629.15 & Eclipsing binary & - \\
\hline
\end{tabular}

$\mathrm{ID}_{\mathrm{BDA}}$ : Identification from the open cluster database by Jean-Claude Mermilliod (http://obswww.unige.ch/webda). 


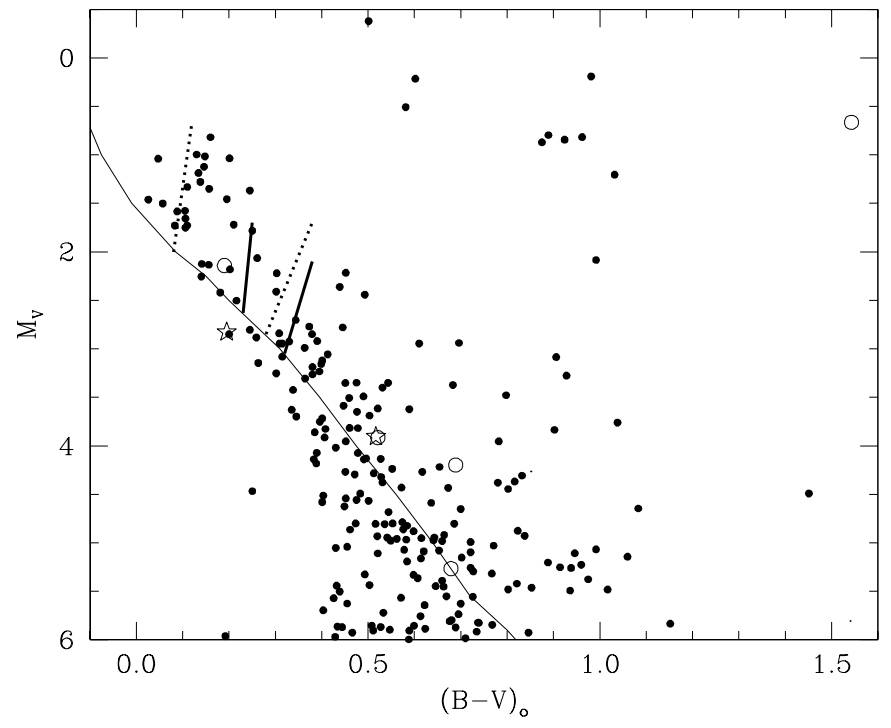

Fig. 8. Positions of seven new variable stars in the color-magnitude diagram of the open cluster NGC 2539. The thin line represents the empirical ZAMS by Sung \& Bessell (1999). The thick dotted and solid bars indicate $\delta$ Scuti (Breger 1979) and $\gamma$ Doradus instability strip (Handler 1999), respectively. Two pulsating stars are marked by star symbols and five eclipsing binary stars by open circles.

candidates in open clusters with older ages than $250 \mathrm{Myr}$; one in M 44 (age 730 Myr, Martín \& Rodríguez 2002), one in Mel 111 (age 450 Myr, Martín 2000) and two in NGC 6633 (age 430 Myr, Martín 2002). Eyer et al. (2002) concluded that recent studies do not show a clear relationship between age and the incidence of $\gamma$ Doradus stars.

\section{Summary}

By performing the $U B V I \mathrm{CCD}$ photometry of the open cluster NGC 2539, we derived a reddening value $E(B-V)=$ $0.06 \pm 0.03$, a distance modulus $\left(V-M_{V}\right)_{0}=10.2 \pm 0.1$ and an age $\log t=8.8$ ( $\sim 630 \mathrm{Myr})$. Seven new variable stars were discovered from the time-series observations in $V$-band. We classify five of them as eclipsing binary stars, one as a $\delta$ Scuti star and one as a possible field $\gamma$ Doradus star.

At present, it might be difficult to make clear what physical parameters such as age (Krisciunas \& Patten 1999) or metallicity (Handler 1999) control $\gamma$ Doradus type variability. A Further photometric survey to search for $\gamma$ Doradus stars in open clusters with various physical parameters would be needed.

Acknowledgements. We appreciate the referee Dr. M. Geffert's valuable comments. We also thank Dr. Y.-J. Moon for his careful reading. This work was partially supported by the Ministry of Science and Technology Research Fund.

\section{References}

Arentoft, T., Sterken, C., Knudsen, M. R., et al. 2001, A\&A, 380, 599

Breger, M. 1979, PASP, 91, 5

Breger, M. 1991, A\&A, 250, 107

Breger, M. 2000, in Delta Scuti and related stars, ed. M. Breger, \& M. H. Montgomery, PASPC, 210, 3

Clariá, J. J., \& Lapasset, E. 1986, ApJ, 302, 656

Cousins, A. W. J., \& Warren P. R. 1963, Mon. Notes Astron. Soc. S. Afr., 22, 65

Dean, J. F., Warren, P. R., \& Cousins, A. W. J. 1978, MNRAS, 183, 569

Eyer, L., Aerts, C., van Loon, M., Bouckaert, F., \& Cuypers, J. 2002, in Observational aspects of pulsating B \& A stars, ed. C. Sterken, \& D. W. Kurtz, PASPC, 256, 203

Frandsen, S., Dreyer, P., \& Kjeldsen, H. 1989, A\&A, 215, 287

Frandsen, S., \& Arentoft, T. 1998, A\&A, 333, 524

Freyhammer, L. M., Arentoft, T., \& Sterken, C. 2001, A\&A, 368, 580

Gilliland, R. L., \& Brown, T. M. 1988, PASP, 100, 754

Girardi, L., Bressan, A., Bertelli, G., \& Chiosi, C. 2000, A\&AS, 141, 371

Handler, G. 1999, MNRAS, 309, L19

Joshi, U. C., \& Sagar, R. 1986, Bull. Astr. Soc. India., 14, 95

Kaye, A. B., Handler, G., Krisciunas, K., Poretti, E., \& Zerbi, F. M. 1999, PASP, 111, 840

Kim, S.-L., \& Lee, S.-W. 1996, A\&A, 310, 831

Kim, S.-L., Chun, M.-Y., Park, B.-G., et al. 2001a, Acta Astron., 51,49

Kim, S.-L., Chun, M.-Y., Park, B.-G., et al. 2001b, A\&A, 371, 571

Krisciunas, K., Crowe, R. A., Luedeke, K. D., \& Roberts, M. 1995, MNRAS, 277, 1404

Krisciunas, K., \& Patten, B. M. 1999, Inf. Bull. Variable Stars, No. 4705

Landolt, A. U. 1973, AJ, 78, 959

Lapasset, E., Clariá, J. J., \& Mermilliod, J.-C. 2000, A\&A, 361, 945

Martín, S. 2000, Ph.D. Thesis, University of Granada, Spain

Martín, S. 2002, private communications

Martín, S., \& Rodríguez, E. 2000, A\&A, 358, 287

Martín, S., \& Rodríguez, E. 2002, in Radial and nonradial pulsations as probes of stellar physics, ed. C. Aerts, T. R. Bedding, \& J. Christensen-Dalsgaard, PASPC, 259, 152

Massey, P., \& Davis, L. E. 1992, A User's Guide to Stellar CCD Photometry with IRAF

Mermilliod, J.-C., \& Mayor, M. 1989, A\&A, 219, 125

Pesch, P. 1961, ApJ, 134, 602

Rodríguez, E., \& Breger, M. 2001, A\&A, 366, 178

Ruprecht, J. 1966, BAICz, 17, 33

Suárez, J.-C., Michel, E., Pérez Hernández, F., et al. 2002, A\&A, 390, 523

Sung, H., \& Bessell, M. S. 1999, MNRAS, 306, 361

Sung, H., Chun, M.-Y., Park, B.-G., et al. 2001, Technical report No. 01-002-039, Korea Astronomy Observatory (in Korean)

Zerbi, F. M., Mantegazza, L., Campana, S., \& Antonello, E. 1998, PASP, 110,804

Zerbi, F. M. 2000, in Delta Scuti and related stars, ed. M. Breger, \& M. H. Montgomery, PASPC, 210, 332 